\title{
Modal properties of toroidal microcavities
}

\author{
S. M. Spillane, T. J. Kippenberg, K. J. Vahala \\ Department of Applied Physics, California Institute of Technology, Pasadena, California 91125 \\ vahala@caltech.edu
}

\begin{abstract}
:
We investigate the resonance structure and modal properties of chip-based toroidal microcavities. The variation of modal volume and radiation quality factor versus the toroidal geometrical aspect ratio is studied.

(C)2004
\end{abstract}

While spherical whispering gallery mode structures possess record quality factors approaching 10 billion [1], they are not readily suited for studies and applications where integration and precise control of their properties is desirable. In order to overcome some of the limitations of spherical resonant structures, toroidal microcavities were developed [2]. Microtoroids retain the ultra-high quality factors representative of microspheres ( $>500$ million has been obtained at $1550 \mathrm{~nm}$ in a toroidal cavity), while possessing distinct advantages due to their ability to be fabricated on a planar silicon substrate. Furthermore, due to the additional degree of freedom in specifying the toroidal geometry, both the number of supported modes and the modal confinement can be dramatically reduced.

However, in contrast to microspheres, toroidal dielectric cavities do not possess closed-form solutions. Nonetheless, access to Q, mode volume, as well as other features of their modes is essential in applications ranging from sensing to nonlinear and quantum optics. In this paper, a finite element eigenvalue method is used to determine the optical fields, frequencies, and radiation quality factor of these structures. By explicitly accounting for the azimuthal degree of symmetry, the eigenvalue problem can be reduced to two dimensions, while preserving the complete vectorial nature of the optical modes.

This numerical method was used to investigate the modes of a toroidal microcavity made of silica. Figure 1 shows the calculated optical field intensity at a resonance wavelength near $1550 \mathrm{~nm}$ for both the fundamental and first transverse modes of a toroidal microcavity with an outer diameter of 50 um and a minor diameter of 6 um. For comparison, the modes of a 50 um diameter microsphere are also given. The figure shows that the optical fields are compressed significantly in a toroidal cavity. Also, in contrast to microspheres, the azimuthal resonant frequencies are no longer degenerate or nearly degenerate. This reduced degeneracy is beneficial in applications to lasing, as the lasing spectrum can now be single-azimuthal (transverse) mode [3].

Fig. 1. Intensity distribution of the fundamental and the first, transverse (azimuthal), TE, optical modes of a toroidal microcavity (left). The minor diameter is $6 \mathrm{um}$ and the outer diameter is $50 \mathrm{um}$. For comparison, a microsphere having the same outer diameter is given on the right. 
The modal volume and radiation limited quality factors have also been investigated. Figure 2 shows the modal volume and radiation limited quality factor for a fundamental mode as a function of the minor toroid diameter. The toroid in the calculation has an outer diameter of $18 \mathrm{um}$ and the resonance wavelength is $852 \mathrm{~nm}$. We see that for weak transverse compressions there is a slow reduction of both mode volume and quality factor, as a result of the additional confinement almost exclusively in the vertical transverse dimension. However, once the transverse compression becomes such that the optical mode is strongly confined in both the radial and vertical directions, the mode approaches that of a fiber and the mode volume and $\mathrm{Q}$ decrease dramatically.
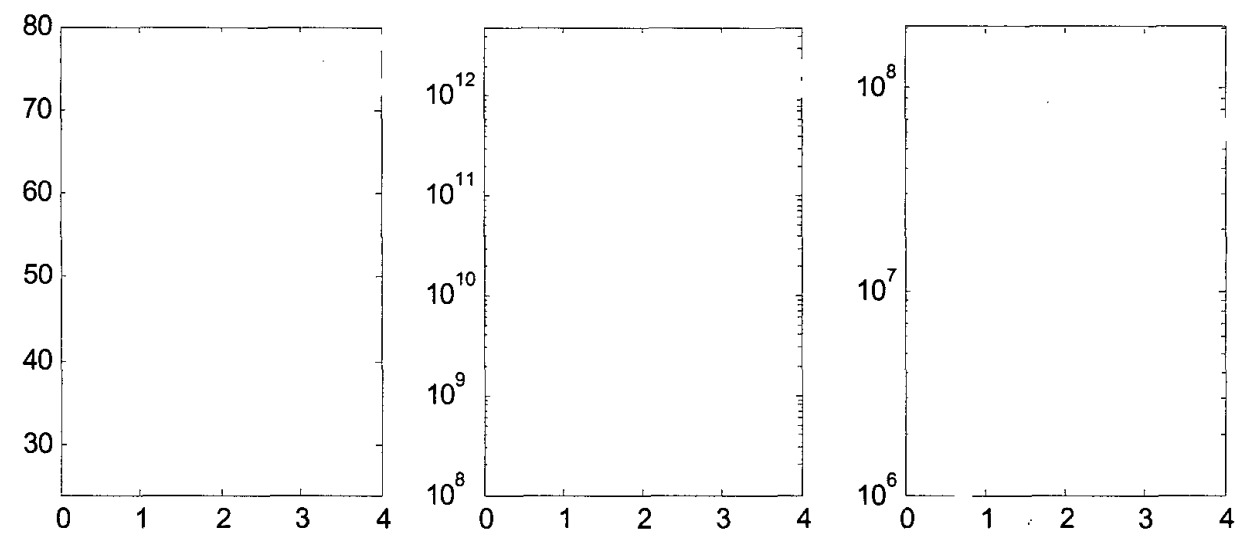

Fig. 2. Cavity mode volume in cubic microns (left) and radiation, quality factor (middle) versus minor, toroid diameter (microns) for a silica microtoroid at a resonance wavelength of $852 \mathrm{~nm}$ with a fixed, outer diameter of 18 microns. TE (TM) modes are illustrated by the circles (squares). The right plot illustrates the ratio of total $\mathrm{Q}$ (combination of radiation and material absorption Q), and mode volume normalized in terms of cubic wavelengths in silica. The data shows a clear maximum of 100 million for a minor toroid diameter of 1.2 micron.

The quantity $\mathrm{Q} / \mathrm{V}$ is a parameter that can be used to characterize microcavities, and is directly related to both the Purcell factor and the critical atom number in cavity quantum electrodynamics [4]. The third panel of figure 2 shows the ratio of overall quality factor to modal volume, normalized such that the mode volume is expressed in terms of cubic wavelengths in the material. The overall quality factor is composed of contributions from radiation loss and material absorption (which is $2.4 \mathrm{e} 10$ at $850 \mathrm{~nm}$ ). Extremely high values of $\mathrm{Q} / \mathrm{V}$ are possible in these structures, with current experimental results at $1550 \mathrm{~nm}$ approaching 2.8 million.

\section{References}

[1] D. W. Vernooy, V. S. Ischenko, H. Mabuchi, E. W. Streed, and H. J. Kimble, "High-Q measurements of fused-silica microspheres in the near infrared" Optics Letters 23, 247-249 (1998).

[2] D. K. Armani, T. J. Kippenberg, S. M. Spillane, and K. J. Vahala, "Ultra-high-Q toroid microcavity on a chip," Nature.421, $925-928$ (2003). [3] S. M. Spillane, T. J. Kippenberg, and K. J. Vahala, "Ultralow-threshold Raman laser using a spherical dielectric microcavity," Nature 415, $621-623(2002)$.

[4] Kerry Vahala, "Optical Microcavities," Nature Insight, 424, 839-846 (2003). 